(Aus dem physiologischen Institut der Universität Leipzig.)

\title{
Zeigen die Aktionsströme verschieden rasch zuckender Muskeln des Frosches einen ver- schiedenen zeitlichen Verlauf?
}

Von

B. P. Babkin (St. Petersburg).

(Mit 2 Textfiguren und Tafel XV.)

Bisher ist noch nie versucht worden, mit den neueren Hilfsmitteln (Registrierung der Aktionsströme durch Kapillarelektrometer und Analyse der erhaltenen Kurven) die für die Auffassung der Aktionsströme des Muskels nicht unwichtige Frage zu beantworten, ob in verschieden rasch zuckenden Muskeln des gleichen Tieres auch die Aktionsströme Verschiedenheiten ihres zeitlichen Ablaufes erkennen lassen. Die einzigen von Le e ${ }^{1}$ ) (1887) gemachten Versuche über Ablauf der Aktionsströme bei den verschiedenen Froschmuskeln können, da gerade der am langsamsten zuckende Hyoglossus nicht verwendet wurde und auch die Methodik für eine genauere Feststellung des Stromverlaufs damals noch nicht zur Verfügung stand, hier übergangen werden. Die Idee, dass Eigentümlichkeiten der Muskelkontraktionen im elektrischen Verhalten zum Ausdruck kommen können, hat aber jener Forscher bereits ausgesprochen.

Auf Veranlassung von Hern Prof. Garten habe ich vor zwei Jahren eine grössere Reihe von Versuchen am Musculus sartorius und hyoglossus (Rana esculenta) angestellt. Den Musculus hyoglossus verwendete ich auf Grund des Hinweises von $\mathrm{Cash}^{2}$ ), dass die Kontraktionsdauer (Dauer des ansteigenden Kurvenastes) cet. par. an diesem Muskel etwa doppelt so lang ist als die des Musculus triceps femoris $(0,205$ Sekunde gegen 0,104 Sekunde). Letzteren

1) Lee, Über die elektrischen Erscheinungen, welche die Muskelzuckungen begleiten. Ludwig's Arbeiten 1887.

2) Cash, Du Bois-Reymond's Arch. 1880 Suppl. S. 147. 
Muskel konnte ich zum Studium der elektrischen Erscheinungen wegen seines schrägen Faserverlaufs nicht gebrauchen und bediente mich statt seiner des parallelfaserigen Musculus sartorius, wenn auch von diesem bekannt ist, dass in ihm neben rasch zuckenden Fasern solche von langsamerer Reaktion vorkommen. Dieser Nacbweis wurde wie bekannt für den Sartorius unter Grützner's Leitung insbesondere von Bonhöffer ${ }^{\imath}$ ) erbracht, der für den Sartorius des Frosches feststellte, dass in ihm eine geringe Überzahl der rasch zuckenden dünnen Fasern vorhanden war (90 dicke Fasern auf 120-110 dünne). Da nun aber der Unterschied der Zuckungskurven des Musculus hyoglossus und sartorius trotz der verschiedenen Fasergattungen in letzteren sehr beträchtlich ist, so konnte man hoffen, an diesen beiden Objekten, wenn sich überhaupt mit der mechanischen Trägheit eine langsamere elektrische Reaktion verknüpft, wenigstens unter den günstigsten Umständen Differenzen im Verlauf der Aktionsströme nachzuweisen.

Die Versuche wurden bei direkter Reizung der betreffenden Muskeln des curaresierten Tieres angestellt und die Muskeln regelmässig nur schwach gespannt, um eine nach einigen Forschern durch verschiedene Belastung möglicherweise eintretende Veränderung des Aktionsstromes auszuschliessen. Die Zwischenstrecke wurde je nach Muskelgrösse etwas verschieden, jedenfalis aber so gross gewählt, dass die durch den Induktionsschlag bedingten Bewegungen des Quecksilbermeniscus die spätere Analyse der Aktionsströme nicht beeinträchtigten. Bei allen entscheidenden Versuchen wurde von Längs- und thermischem Querschnitt abgeleitet, da namentlich bei kleiner Elektrometerstrecke das frühe Auftreten der zweiten Phase für die Frage nach dem Erregungsvorgang unter der Längsschnittelektrode schwer entwirrbare Verhältnisse schafft ${ }^{2}$ ).

Ferner war die Zwischenstrecke bei dem Musculus hyoglossus

1) Bonhöffer, Pflüger's Arch. Bd. 47 S. 125. 1889.

2) Hierbei bleibt natürlich die Frage unerörtert, ob nach Verletzung des einen Muskelendes die erste Phase des Aktionsstromes, die hier dann allein auftritt, einen anderen Verlauf besitzt als die erste Phase des zweiphasischen Aktionsstromes, wie er am unverletzten Muskel zu beobachten wäre. Da Musculus sartorius und hyoglossus der gleichen Schädigung ausgesetzt wurden, so liegt kein Grund vor, die Querschnittsanlegung für den unten zu schildernden verschiedenartigen Verlauf des einphasischen Aktionsstromes bei beiden Muskeln verantwortlich zu machen. 
Zeigen die Aktionsströme verschieden rasch zuckender Muskeln etc. 597

meist kleiner als bei dem Musculus sartorius. Wäre aber eine scheinbare Verlangsamung der elektrischen Erscheinungen im ausgeschnittenen Muskel durch verschieden rasche Leitung in den einzelnen Fasergruppen bedingt, so hätte man bei der grösseren Zwischenstrecke am Sartorius viel eher eine Verlängerung des elektrischen Vorgangs erwarten müssen. Die kürzere Zwischenstrecke am Hyoglossus würde also a fortiori dafür sprechen, dass nicht jene Ursache in Betracht kommt.

Schon meine ersten, zum Teil bei höherer Temperatur ausgeführten Versuche ergaben mir das Resultat, dass der Verlauf des Aktionsstromes im Musculus sartorius ein rascherer ist und auch die Leitungsgeschwindigkeit in diesem Muskel unter sonst gleichen Bedingungen die im Museulus hyoglossus übertrifft. Finwandsfrei lassen sich die genannten Unterschiede aber erst dadurch demonstrieren, dass man in beiden Muskeln durch Abkühlung auf ganz die gleiche Temperatur den Ablauf des Erregungsvorgangs zeitlich in die Länge zieht. Auf die gleichzeitige graphische Registrierung der Zuckungskurven habe ich bei den letztgenannten Versuchen verzichtet, doch wurden nachträglich, um die verschieden rasche mechanische Reaktion der beiden Muskeln bei der Abkühlung auch darzutun, Zuckungskurven von ihnen unter isotonischen Bedingungen bei der gleichen Aussentemperatur auf der berussten Trommel aufgenommen.

Nachstehende Kurven (Fig. 1 und 2) zeigen die. Kontraktion des Musculus sartorius und des Musculus hyoglossus bei einer Temperatur von $+4{ }^{0}$ C. Während beim Sartorius in etwa 0,43 Sekunden der Gipfel erreicht ist, verstreicht beim Musculus hyoglossus eine Zeit von etwa 0,70 Sekunden bis zum Zuckungsmaximum. Ähnliches Verbalten boten auch andere, bei jener 'Temperatur aufgenommene Kurven dar. Es wäre also das Verhältnis der Gipfelzeiten etwa gleich $4: 7^{1}$ ).

Das Verhalten der Aktionsströme wird durch bẹistehende zwei Kurvenpaare demonstriert, die hier in zwei Drittel der Originalgrösse wiedergegeben sind. Die Fig. 3 und 4 Taf. XV zeigen den einphasischen Aktionsstrom des Musculus sartorius (Fig. 3) und des

1) Die angeführten Kurven wurden aus äusseren Gründen nach meiner Abreise von Dr. N. P. Tichomirow aufgenommen, dem ich für seine Bemühungen auch an dieser Stelle noch bestens danken möchte. 
Musculus byoglossus (Fig. 4) bei der gleichen Temperatur $\left(+3^{0}\right)$. Die weissgezeichnete analysierte Kurve ist nach dem von Garten ${ }^{1}$ ) angegebenen Verfahren erhalten. Fig. 3 zeigt uns, dass der Anstieg beim Musculus sartorius in 3,4 ganzen Schwingungen der zur Registrierung der Ordinaten benutzten Zungenpfeife rollendet ist (Abstand einer Ordinate von jeder übernächsten gleich $1 / 258$ Sekunde), während beim Museulus hyoglossus (Fig. 4) die Anstiegzeit 5,2

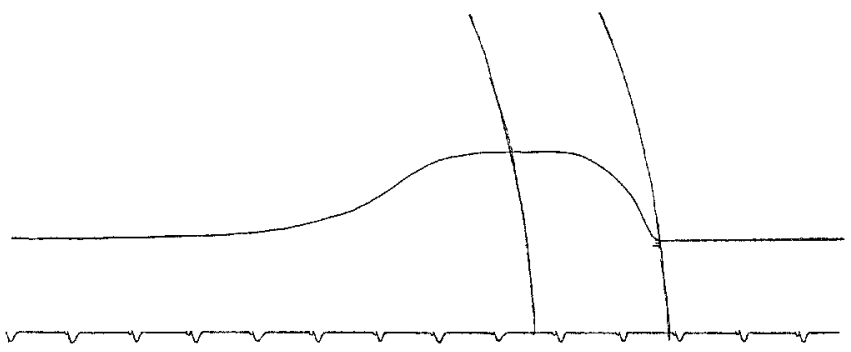

Fig. 1. Sartorius, direkte Reizung, 1 Akk., R.-A. $=68 \mathrm{~mm} .12$ Min. abgekühlt. Kammertemperatur $+4^{0}$ C. Hebelvergrösserung 1:9.

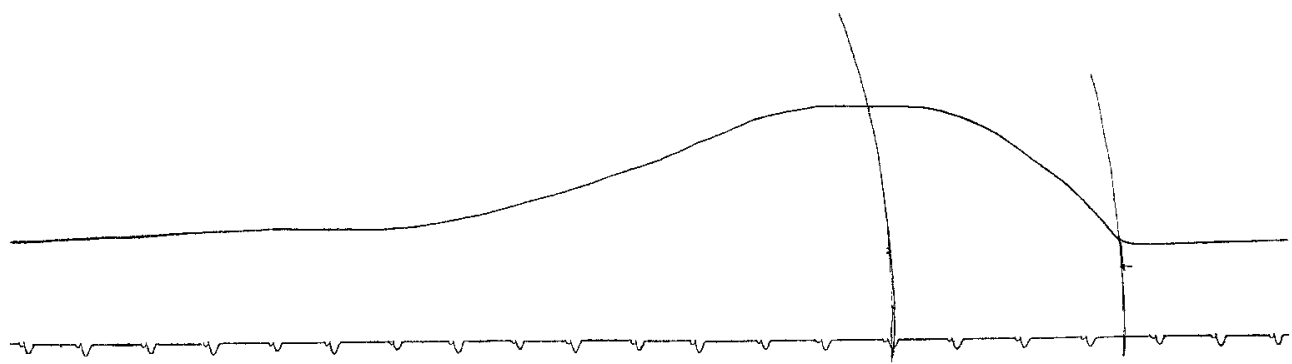

Fig. 2. Hyoglossus. 10 Min. abgekühlt. Kammertemperatur $=+4^{\circ}$ C. R.-A. $=61 \mathrm{~mm}$. Sonst alles wie oben.

ganze Schwingungen dauert. Es entsprächen diese Anstiegszeiten 13 bzw. $20 \sigma$, und das Verhältnis derselben wäre nahezu $2: 3$.

Bei den anderen beiden abgebildeten Versuchen (Taf. XV Fig. 5 und 6) betrug die Temperatur $3,6^{\circ}$, und hier ist die Anstiegzeit bereits wesentlich kleiner: beim Sartorius (Fig. 5) 9,7 $\sigma$, beim Hyoglossus (Fig. 6) 13,1 o. Da in der Nähe des Nullpunktes so kleine Temperaturunterschiede bei beiden Muskeln so auffallende Differenzen in der Anstiegszeit des Aktionsstromes geben, so wurde

1) Garten, Ein einfaches Verfahren zur Ausmessung der Kapillarelektrometerkurven. Pflüger's Arch. Bd. 89 S. 613. 1902. 
Zeigen die Aktionsströme verschieden rasch zuckender Muskeln etc. 599

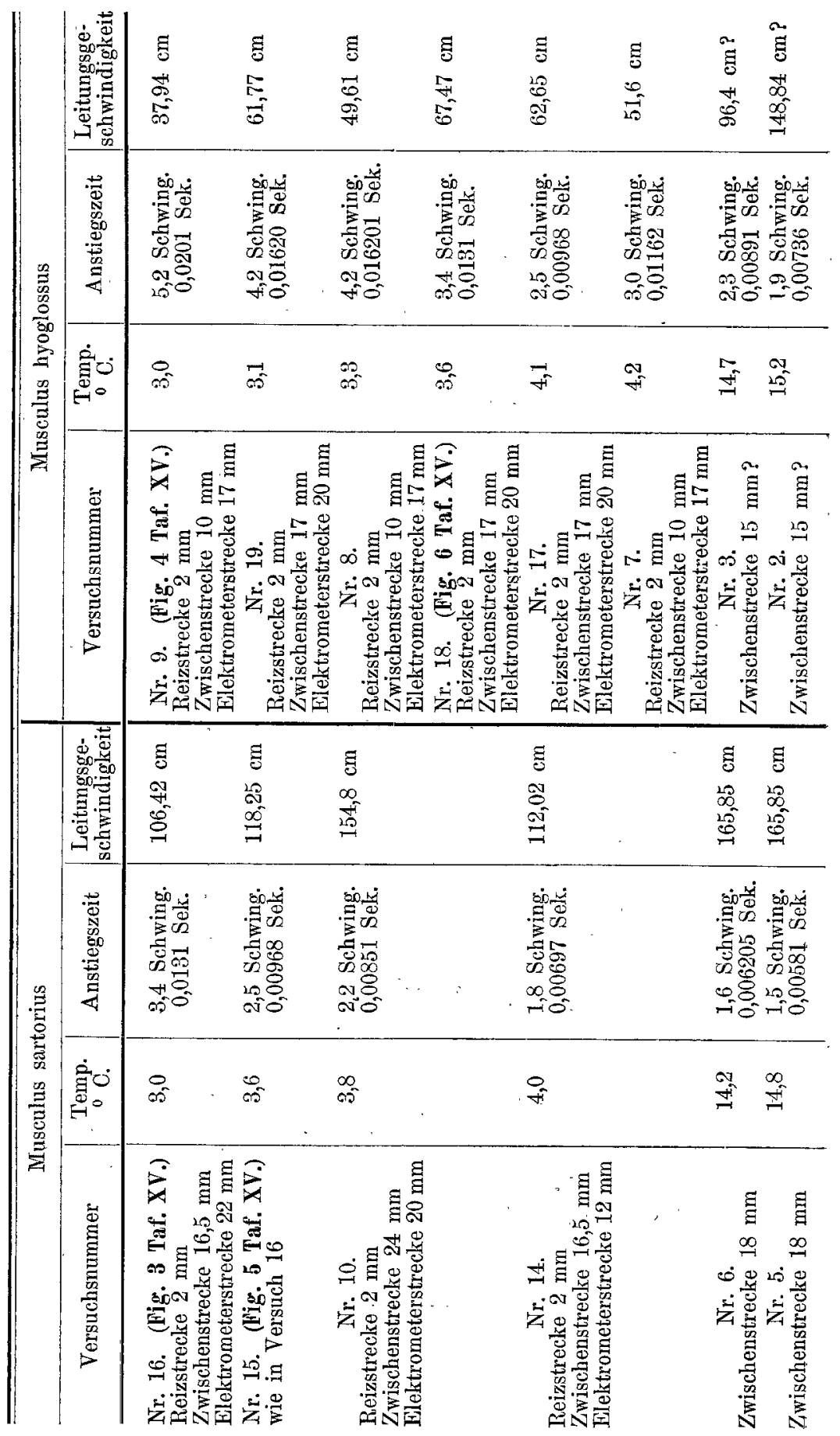


auf genaue Temperaturablesung besonders geachtet und durch Häufung der Versuche Zufälligkeiten möglichst ausgeschlossen. Ohne hier auf die diesbezüglichen älteren Versuche, die mir aber das gleiche Resultat ergeben haben, näher einzugehen, seien hier nur die letzten Versuchsergebnisse, bei denen in der Hauptsache bei niedrigerer Temperatur gearbeitet wurde, in vorstehender Tabelle kurz angeführt. Ausser der Anstiegszeit ist unter der Annahme des Fehlens einer elektrischen Latenz aus der Zwischenstrecke und der Zeit zwischen Reizmoment und Schwankungsbeginn die Leitungsgeschwindigkeit berechnet. Es ergibt sich in allen Versuchen übereinstimmend, bis auf den Versuch bei $14,7^{\circ}$, wo der protokollierte Zahlenwert für die Zwischenstrecke nicht sicher zu lesen war, eine beträchtlich grössere Leitungsgeschwindigkeit der Erregung im Musculus sartorius als im Musculus hyoglossus. Aus älteren Versuchen sei hier summarisch nur noch angeführt, dass der Hyoglossus bei einer Durchschnittstemperatur von $9,83^{\circ}$ eine Anstiegsdauer des Aktionsstromes von 0,0088 Sekunden ergab und der Musculus sartorius, trotz etwas niedrigerer Temperatur $\left(9-8^{\circ}\right)$ eine kürzere Anstiegsdauer des Aktionsstromes besass; dieselbe betrug 0,0063 Sekunden. Die genannten Werte sind der Durchschnitt von fünf bzw. vier Versuchen.

Durch die angeführten Versuche ist der Nachweis erbracht, dass der langsamer zuckende Musculus hyoglossus auch in bezug auf seine elektrischen, den Erregungsvorgang begleitenden Erscheinungen träger reagiert als der gleich temperierte Musculus sartorius. Da aber die Unterschiede in absoluten Zeitwerten relativ klein sind, so ist der Nachweis hierfür, wenn die Registriermethoden nicht noch weiter vervollkommnet werden, nur nach gleichmässiger Abkühlung beider Muskeln wie in dem angeführten Beispiel mit voller Sicherheit $\mathrm{zu}$ erbringen.

Zum Schluss möchte ich Herrn Geheimrat Hering noch bestens danken für das rege Interesse an meiner Arbeit sowie den Herren Prof. Garten, Dr. v. Brücke und Dr. Dittler für die Hilfe, die sie mir jederzeit angedeihen liessen. 


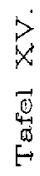
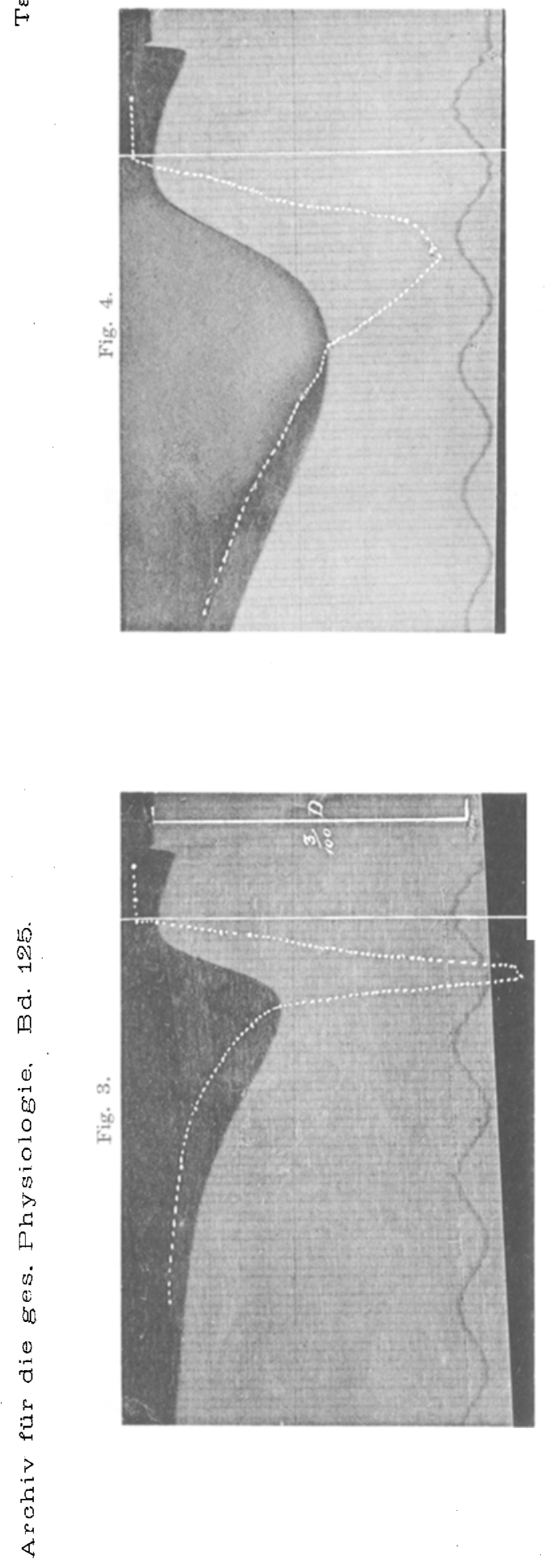
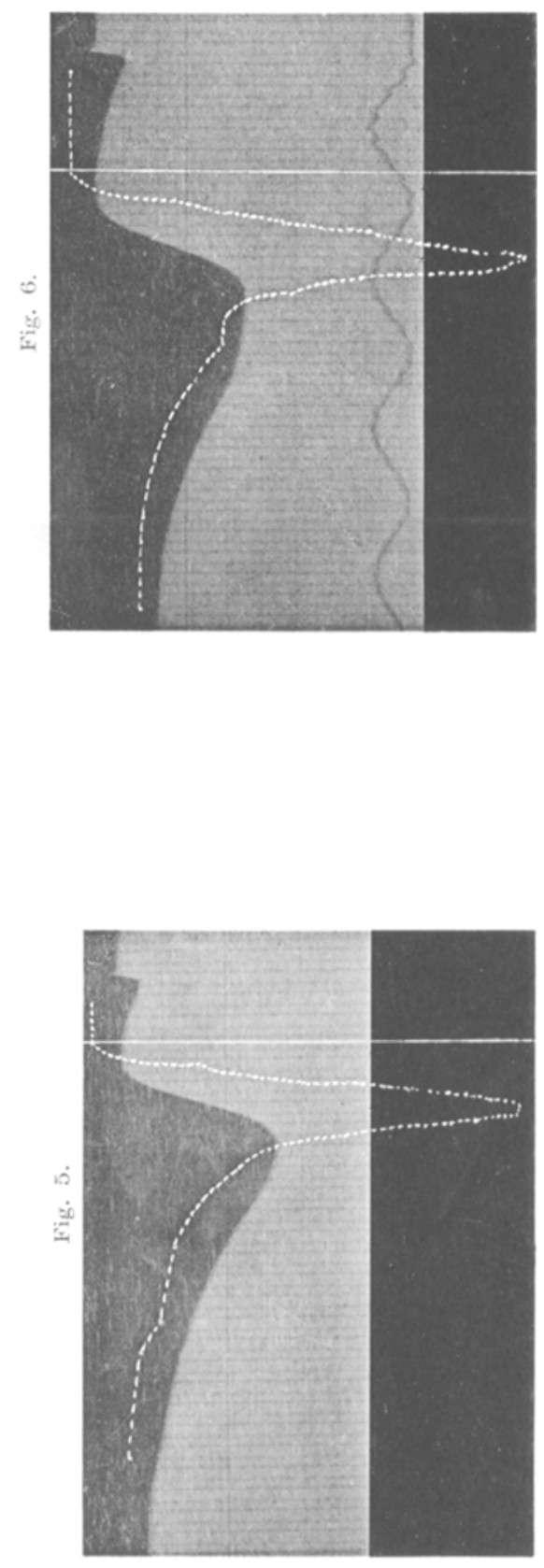

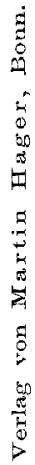

